\title{
Effects of Several Beta-Blockers on Hemodynamics of SHR
}

\author{
Takashi Sasaki, Nagao Kajiwara, Masayuki Koshino, \\ Gentaro Takahashi, Masahiko Kogure, Minoru Kubota, \\ Akio Yamamura, Muneaki Furukata, Kazuaki Kuroda, \\ Katsuichiro Sakai, Hirobumi Ueda, and Michinobu Hatano
}

We have reported about the effects of $\alpha$ - and $\beta$-adrenergic blockers on hemodynamics of spontaneously hypertensive rats (SHR), and summarised that $\beta$-blocker (propranolol $50 \gamma$ ) depressed cardiac output, but increased blood pressure and systemic vascular resistance. The experiment has been designed to know the effects and the relationships of doses of several beta-blockers on the hemodynamics of SHR.

\section{Materials and Methods:}

SHR and WR (normotensive Wistar rats) weighing 180-230 Gm were used in this experiment. Under light ether anesthesia, $\alpha$-chloralose and gallamine were added, and the aeration was maintained by the artificial respiration. The chest was opened, an electromagnetic flow probe was placed around the thoracic aorta for measuring cardiac output (COP). A catheter was inserted from the femoral artery, advanced to the central aorta for measuring of blood pressure (BP). Left ventricular pressure (LVP) was recorded through the catheter inserted into the left ventricular cavity by direct puncturing. BP, COP, LVP, LVP dp/dt, systemic peripheral vascular resistance (SVR), and heart rate were measured simultancously, before and after the infusion of drugs. Practolol (Eraldin) as $\beta_{\mathbf{1}}$-blocker, H35/25 as $\beta_{2}$-blocker, and propranolol (Inderal) were used. Each drug was administered via a catheter inserted to the jugular vein. Initial dose of $25 \mathrm{mg}$ was followed by $50 \gamma, 100 \gamma$, and $200 \gamma$ at about $10 \mathrm{~min}$ intervals.

\section{Results:}

In SHR, BP was increased with propranolol at doses of $25 \gamma$ and $50 \gamma$, but was decreased at doses of $100 \gamma$ and $200 \gamma$. The similar tendency was seen in WR, but the response was greater in SHR. After practolol or $\mathbf{H 3 5 / 2 5}$ injection at doses of $100 \gamma$ and $200 \gamma, \mathrm{BP}$ was increased but the response was greater at $100 \gamma$ than at $200 \gamma$, in SHR. In WR, BP was increased at a dose of $50 \gamma$, but the response was less than in SHR. It may be said that the sympathetic tone is easily accelerated in SHR. COP was decreased by propranolol at $50 \gamma$ and was increased at $100 \gamma$ and $200 \gamma$ in SHR. The effect was similar in WR. COP was decreased by practolol or $\mathrm{H} 35 / 25$ at $100 \gamma$ and was increased at $200 \gamma$. Although COP was increased, BP, LVP, and LVP dp/dt were decreased. Therefore it is considered that the increment of COP was caused by a diminished after load. SVR was increased by propranolol at $50 r$ and was decreased at $100 \mathrm{r}$ and $200 \mathrm{r}$ in SHR.

From the Second Department of Internal Medicine (Department of Cardiology, Surugadai Nihon University Hospital), Nihon University School of Medicine, Tokyo. 
There was a similar tendency in WR but the response was greater in SHR. SVR was increased by practolol or $\mathrm{H} \mathrm{35/25}$ at $50 \gamma$ and $100 \gamma$ in both SHR and WR, but was decreased at $200 \gamma$ in WR. These results indicate that the sympathetic tone is easily accelerated in SHR. Bradycardia was induced by propranolol at $50 \gamma$, and practolol or $\mathrm{H} 35 / 25$ at $100 \gamma$ both in SHR and WR.

Conclusion:

We have reported that there was a hypersensitivity to beta blockers in SHR and the above mentioned results seem to support our opinion. Considering that similar changes were seen with practolol and $\mathrm{H} 35 / 25$, probably it is impossible to make a quotion from the results of SHR to human being or dog. The above results could be explained as the species specific reaction and the dose dependent responses. 